\title{
Neural Network Based Real-time Correction of Transducer Dynamic Errors
}

\author{
J. Roj \\ Institute of Measurement Science, Electronics and Control, Silesian University of Technology, Gliwice, Poland, \\ jerzy.roj@polsl.pl
}

In order to carry out real-time dynamic error correction of transducers described by a linear differential equation, a novel recurrent neural network was developed. The network structure is based on solving this equation with respect to the input quantity when using the state variables. It is shown that such a real-time correction can be carried out using simple linear perceptrons. Due to the use of a neural technique, knowledge of the dynamic parameters of the transducer is not necessary. Theoretical considerations are illustrated by the results of simulation studies performed for the modeled second order transducer. The most important properties of the neural dynamic error correction, when emphasizing the fundamental advantages and disadvantages, are discussed.

Keywords: Artificial neural network, linear perceptron, dynamic errors correction, measuring transducer, state variables.

\section{INTRODUCTION}

$\mathrm{T}$ HE PROCESSING taking place in transducers used for digital measurements of instantaneous values of timevarying quantities generally consists of several phases $[1,2]$. First, the measured input quantity is converted to an electrical signal (usually voltage or current). For this purpose appropriate sensors and signal conditioning circuits are used. Then, sampling and quantization are carried out to obtain digital values proportional to the instantaneous values of the output quantities of the analog measuring chain. In the last phase digital processing enabling obtaining sufficiently accurate measurement results of the input quantity at discrete moments of time is performed.

Digital processing, usually carried out by a microprocessor, consists of performing the activities determined by appropriate static and dynamic error correction algorithms whose properties co-decide about the accuracy of the whole measuring chain. Implementation of this kind of operations assumes the knowledge of the inverse numerical model describing the measuring process from its input to the A/D converter output [3].

In many cases, sufficiently accurate identification of the inverse analog processing model is very complex or even impossible to be carried out. An alternative approach is to replace selected algorithms or algorithm chains by artificial neural networks (ANN) which, in the learning process, can "create" a right inverse model $[4,5,6]$ due to their basic features: the ability to learn and to generalize $[7,8,9]$. The ANN properties of this type appear to be significant for the issues of correction of dynamic errors which are usually caused by the dynamic properties of sensors [10, 11]. Classical dynamic error correction algorithms are usually characterized by high complexity of numerical operations, in particular in the case of describing the transducer dynamics by means of higher order differential equations.

ANN as "universal approximators" $[12,13,14]$ have been widely used for transducer static error correction $[15,16,17$, $18]$, in particular for transducer and measuring instrument calibration $[19,20,21]$. Nevertheless, in the field of real- time dynamic error correction, solutions using DSP [22, 23, 24], FPGA technique [25] and analog circuits [26, 27] are dominant. The typical use of ANN is presented, e.g., in [11, 28, 29]. In these publications MLP with tapped delay lines and RBF networks have been used for the dynamic error correction. The network input data is usually from a few to hundreds of the time series samples of the sensor step response, while the predicted values in steady state are obtained at the network output.

The author presents a different approach to such issues. It is based on using a relatively simple recurrent neural network for solving (with respect to the input quantity) the differential equation describing the dynamic properties of a transducer $[5,6]$. This allows for a fast performance of the real-time dynamic error correction, described in this paper.

\section{Methodology}

Dynamic properties of analog transducers are usually modeled by a linear differential equation of $n$-th order [ 30 , 31, 32]:

$$
y^{(n)}+a_{n-1} y^{(n-1)}+\ldots+a_{1} \dot{y}+a_{0} y=b_{0} x
$$

where: $a_{n-1}, \ldots, a_{0}, b_{0}$ are constant coefficients, $x$ is the input quantity, $y$ is the transducer output quantity. Relationship (1) can be written in the form of $n$ state equations $[33,34,35]$ :

$$
\begin{aligned}
& \dot{y}_{1}=y_{2}, \\
& \dot{y}_{2}=y_{3}, \\
& \quad \vdots \\
& \dot{y}_{n-1}=y_{n}, \\
& \dot{y}_{n}=-2 a_{n-1} y_{n}-\ldots-a_{0} y_{1}+b_{0} x,
\end{aligned}
$$

where $y_{1}, \ldots, y_{n}$ are state variables, and $y_{1}=y$.

The set of equations (2) can be given in the matrix form: 


$$
\dot{y}=F \boldsymbol{y}+G x,
$$

where:

$$
\boldsymbol{y}=\left[\begin{array}{l}
y_{1} \\
y_{2} \\
\vdots \\
y_{n-1} \\
y_{n}
\end{array}\right], \boldsymbol{F}=\left[\begin{array}{cccc}
0 & 1 & \ldots & 0 \\
0 & 0 & \ldots & 0 \\
\vdots & \vdots & \ldots & \vdots \\
0 & 0 & \ldots & 1 \\
-a_{0}-a_{1} & \ldots & -a_{n-1}
\end{array}\right], \boldsymbol{G}=\left[\begin{array}{c}
0 \\
0 \\
\vdots \\
0 \\
b_{0}
\end{array}\right] .
$$

The solution of (3) is the relation:

$$
\boldsymbol{y}\left(t_{k+1}\right)=e^{\boldsymbol{F} T_{d}} \boldsymbol{y}\left(t_{k}\right)+e^{\boldsymbol{F} T_{d}} \int_{t_{k}}^{t_{k+1}} e^{-\boldsymbol{F} \tau} \boldsymbol{G} x(\tau) d \tau,
$$

where $T_{d}=t_{k+1}-t_{k}$ is the sampling period. Assuming that the values of $y$ do not change between the sampling points (changes of $y$ occur only at the moments of discretization), relationship (3) can be written as the discrete state equation:

$$
\boldsymbol{y}(k+1)=\boldsymbol{\Phi} \cdot \boldsymbol{y}(k)+\boldsymbol{\Psi} \cdot x(k),
$$

where:

$$
\boldsymbol{y}(k)=\left[\begin{array}{c}
y_{1}(k) \\
\vdots \\
y_{n}(k)
\end{array}\right]
$$

and

$$
\begin{aligned}
& \boldsymbol{\Phi}=e^{F T_{d}}=\left[\begin{array}{ccc}
\varphi_{11} & \ldots & \varphi_{1 n} \\
\vdots & \\
\varphi_{n 1} & \ldots & \varphi_{n n}
\end{array}\right], \\
& \boldsymbol{\Psi}=\boldsymbol{G} \int_{0}^{T_{d}} e^{\boldsymbol{F}\left(T_{d}-\tau\right)} d \tau=\left[\begin{array}{c}
\psi_{1} \\
\vdots \\
\psi_{n}
\end{array}\right] \text {. }
\end{aligned}
$$

Matrix equation (6) can be presented in the form of the following equations:

$$
\begin{aligned}
& y_{1}(k+1)=\varphi_{11} y_{1}(k)+\ldots+\varphi_{1 n} y_{n}(k)+\psi_{1} x(k), \\
& y_{2}(k+1)=\varphi_{21} y_{1}(k)+\ldots+\varphi_{2 n} y_{n}(k)+\psi_{2} x(k), \\
& \quad \vdots \\
& y_{n}(k+1)=\varphi_{n 1} y_{1}(k)+\ldots+\varphi_{n n} y_{n}(k)+\psi_{n} x(k) .
\end{aligned}
$$

Taking into account that the state variable $y_{1}$ is directly measured (there is $y_{1}=y$ ) and solving equations (10) with respect to the input quantity $x$, the following recurrent equations are obtained $[6,35]$ :

$$
\hat{x}(k)=\frac{1}{\psi_{1}}\left[-\varphi_{11} y(k)-\varphi_{12} \hat{y}_{2}(k)-\ldots-\varphi_{1 n} \hat{y}_{n}(k)+y(k+1)\right],
$$

and

$$
\begin{aligned}
& \hat{y}_{2}(k+1)=\varphi_{21} y(k)+\varphi_{22} \hat{y}_{2}(k)+\ldots+\varphi_{2 n} \hat{y}_{n}(k)+\psi_{2} \hat{x}(k), \\
& \vdots \\
& \hat{y}_{n}(k+1)=\varphi_{n 1} y(k)+\varphi_{n 2} \hat{y}_{2}(k)+\ldots+\varphi_{n n} \hat{y}_{n}(k)+\psi_{n} \hat{x}(k),
\end{aligned}
$$

where $\hat{x}(k)$ is the estimate of the input quantity $x$ at moment $k$, obtained from measurement of the output quantity $y$ at moments $k+1, k$ (denoted as $y(k+1), y(k)$ ) and the estimates of the state variables $\hat{y}_{2}(k), \ldots, \hat{y}_{n}(k)$ from the previous moment. Equations (11) and (12) represent the numerical solution (with respect to the input quantity) of the differential equation describing the transducer dynamic properties. Thus, they enable performing the real-time dynamic error correction.

\section{NEURAL NETWORK TECHNIQUE FOR THE REAL-TIME DYNAMIC ERROR CORRECTION}

Equations (11) and (12) can be written as:

$$
\hat{x}(k)=\sum_{i=2}^{i=n} w_{1 i} \hat{y}_{i}(k)+w_{11} y(k)+w_{10} y(k+1)
$$

and

$$
\begin{aligned}
& \hat{y}_{2}(k+1)=\sum_{i=2}^{i=n} w_{2 i} \hat{y}_{i}(k)+w_{21} y(k)+w_{20} \hat{x}(k), \\
& \vdots \\
& \hat{y}_{n}(k+1)=\sum_{i=2}^{i=n} w_{n i} \hat{y}_{i}(k)+w_{n 1} y(k)+w_{n 0} \hat{x}(k),
\end{aligned}
$$

where the coefficients $w_{i j}$ have constant values and are functions of $\varphi_{i j}$ and $\psi_{i}$. The operations described by (13) and (14) can be carried out using simple linear perceptrons, connected recurrently in a network structure shown in Fig.1.

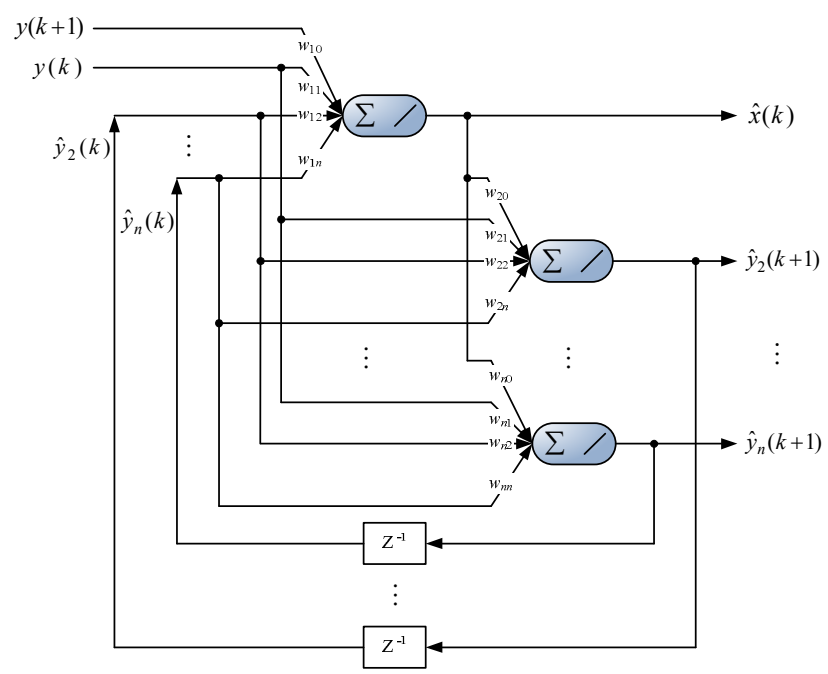

Fig.1. Structure of the recurrent neural network performing realtime dynamic error correction according to equations (13) and (14). 
In this case, the real-time dynamic error correction can be illustrated by a sequence of operations shown in Fig.2.

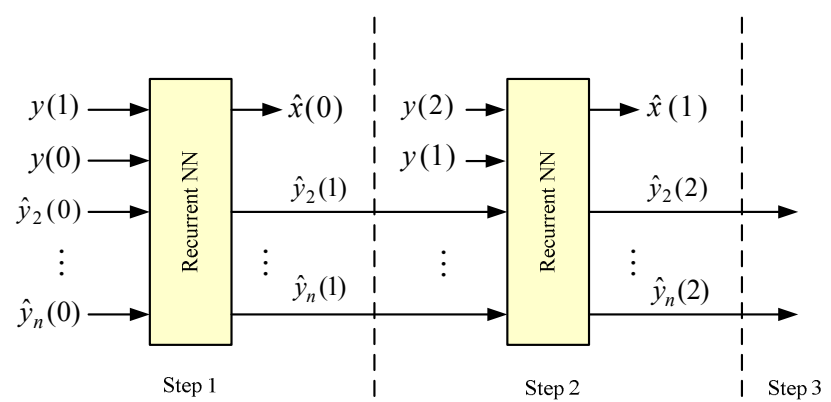

Fig.2. Illustration of dynamic error correction performance using neural network structure shown in Fig.1.

In the first step of the dynamic error correction, knowledge of the initial values of state variables $\hat{y}_{2}(0), \ldots, \hat{y}_{n}(0)$ is required. These values can be estimated or assumed to be equal to zero. The use of initial values different from the actual values will result in a transient state, which disappears after a certain number of the correction steps [6].

In the case of the first-order transducer model:

$$
\dot{y}+a_{0} y=b_{0} x,
$$

equation (11) takes the non-recurrent form:

$$
\hat{x}(k)=\frac{1}{\psi}[-\varphi y(k)+y(k+1)]=w_{1} y(k)+w_{2} y(k+1),
$$

where: $w_{1}=-\frac{\varphi}{\psi}$, and $w_{2}=\frac{1}{\psi}$.

It means that for such a case the dynamic error correction is possible to be carried out with use of a simple, two-input linear perceptron, as shown in Fig.3.

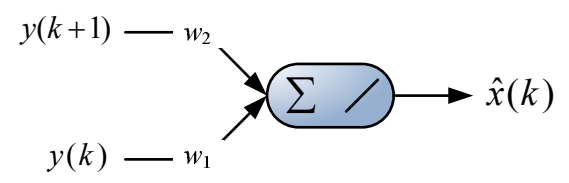

Fig.3. Linear perceptron performing dynamic error correction in the case of the sensor model described by a first order linear differential equation (15).

If the dynamic model of a transducer is described by the second-order differential equation:

$$
\ddot{y}+a_{1} \dot{y}+a_{0} y=b_{0} x,
$$

formulas (11) and (12) take the form:

$$
\hat{x}(k)=\frac{1}{\psi_{1}}\left[y(k+1)-\varphi_{11} y(k)-\varphi_{12} \hat{y}_{2}(k)\right],
$$

$$
\hat{y}_{2}(k+1)=\varphi_{21} y(k)+\varphi_{22} \hat{y}_{2}(k)+\psi_{2} \hat{x}(k),
$$

where $\hat{x}(k)$ is the estimate of the input quantity $x$ at moment $k, \hat{y}_{2}(k)$ is the estimate of the state variable $y_{2}$.

Equations (18) and (19) can be written as:

$$
\begin{aligned}
& \hat{x}(k)=w_{1} y(k+1)+w_{2} y(k)+w_{3} \hat{y}_{2}(k), \\
& \hat{y}_{2}(k+1)=v_{1} y(k)+v_{2} \hat{y}_{2}(k)+v_{3} \hat{x}(k),
\end{aligned}
$$

where the coefficients $w_{1} \ldots w_{3}$ and $v_{1} \ldots v_{3}$ have constant values and are functions of $\varphi_{11} \ldots \varphi_{22}, \psi_{1}, \psi_{2}$.

The operations described by (20) and (21) can be carried out using simple linear perceptrons with 3 inputs, connected as shown in Fig.4.

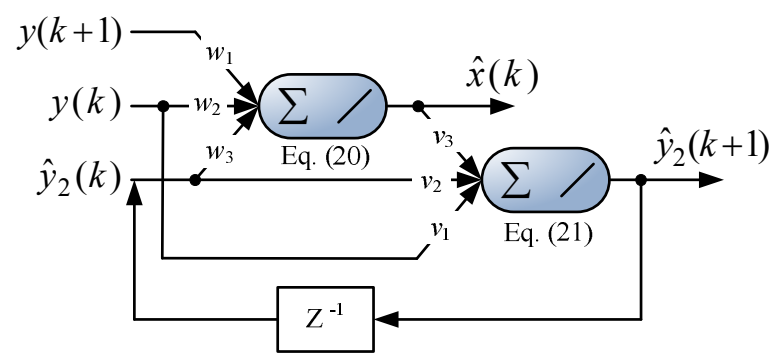

Fig.4. Structure of the recurrent linear perceptron network performing dynamic error correction in the case of the sensor model described by a second order linear differential equation (17).

There is also possible non-recurrent performing of the dynamic error correction by a single perceptron shown in Fig.5. and functioning in accordance with (20).

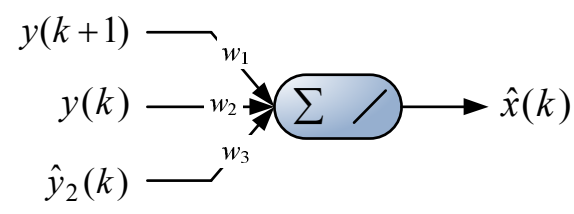

Fig.5. Linear perceptron performing non-recurrent dynamic error correction.

In this case, it is necessary to determine the values of state variable $\hat{y}_{2}$ by, e.g., using the so-called central difference:

$$
\hat{y}_{2}(k)=\frac{y(k+1)-y(k-1)}{2 T_{\mathrm{d}}},
$$

where: $T_{d}=t_{k+1}-t_{k} \quad$ is the sampling period, $y(k+1)=y\left(t_{k}+T_{\mathrm{d}}\right), y(k-1)=y\left(t_{k}-T_{\mathrm{d}}\right)$.

During the correction, the values of $\hat{y}_{2}(k)$ are calculated online, at each step. 


\section{Simulation RESUlTS}

Investigations of possibilities of using an ANN for the real-time dynamic error correction were carried out with use of the MATLAB Neural Toolbox library [36]. The case of the first-order transducer is trivial, so the second-order transducer was the object of studies. Its dynamic properties are usually modeled by the linear differential equation [30, 31]:

$$
\ddot{y}+2 \xi \omega_{0} \dot{y}+\omega_{0}^{2} y=\omega_{0}^{2} k_{s} x
$$

where: $\xi$ is the damping coefficient, $\omega_{0}$ - natural frequency, $k_{s}$ - static gain.

To perform preliminary investigations, the following values of the transducer parameters were assumed: the damping coefficient $\xi=0.5$, the natural frequency $\omega_{0}=10 \mathrm{rad} / \mathrm{s}$ and the static gain $k_{s}=1$. So the chosen parameter values characterized a second-order underdamped transducer. It was also assumed that the sampling period $T_{d}=0.02 \mathrm{~s}$.

The ANN learning process requires a set of learning patterns $[7,8]$. This set was generated using the dependence describing the transducer response for the step input quantity changing from the value $x_{0}$ to $x_{1}[30,31]$ :

$$
y(t)=\left(x_{1}-x_{0}\right)\left[1-\frac{e^{-\xi \omega_{0} t}}{\sqrt{1-\xi^{2}}} \sin (\omega t+\theta)\right]+x_{0},
$$

where

$$
\omega=\omega_{0} \sqrt{1-\xi^{2}}
$$

and

$$
\theta=\operatorname{arctg}\left(\frac{\omega}{\xi \omega_{0}}\right)
$$

Each element of the first perceptron (performing equation (20)) learning pattern set should therefore consist of vector $\boldsymbol{i n}_{i j}^{a}=\left[y_{i j}\left(t_{j}+T_{d}\right), y_{i j}\left(t_{j}\right), \hat{y}_{2 i j}\left(t_{j}\right)\right]^{\mathrm{T}}$ fed to the perceptron input and values out $_{i}^{a}=x_{1 i}$ (for which there were determined $\boldsymbol{i n}_{i j}^{a}$ ) fed to its output. The index $i$ represents a subsequent input step, $j-$ a subsequent time moment. Similarly, for the second perceptron, performing $(21)-$ the vector of the input values is given by $\boldsymbol{i n}_{i j}^{b}=\left[y_{i j}\left(t_{j}\right), \hat{y}_{2 i j}\left(t_{j}\right), x_{1 j}\right]^{\mathrm{T}} \quad$ and $\quad o u t_{i}^{b}=\hat{y}_{2 i j}\left(t_{j}+T_{d}\right)$. Preparation of this kind of learning sets requires, among other things, the knowledge of subsequent state variable values $\hat{y}_{2 i j}$, that is, the determination of derivatives. For the purpose of simulations, the derivatives were determined using relationship (22).

It was also assumed that the measured quantity $x(t)$ was changed stepwise as shown in Fig.6. (at first the step change from 0 to 0.8 , and then from 0.8 to 0.2 ).

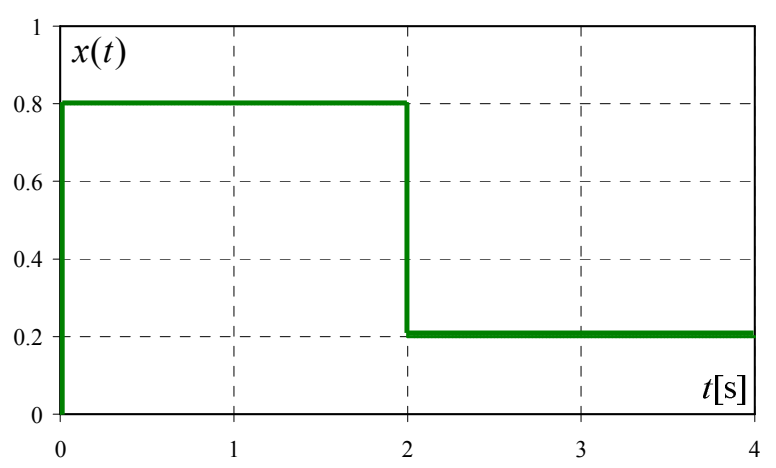

Fig.6. The measured quantity $x(t)$.

In the learning process the Levenberg-Marquardt algorithm [9, 37] and 200-element learning sets obtained from equations (22) and (24) were used. To verify the learning quality of both perceptrons, the testing procedure with the 200000 -element testing set whose values were generated in the same way as those of the learning set was carried out.

It should be noted that during the recurrent implementation of the dynamic correction by the network from Fig.4., the initial value of state variable $\hat{y}_{2}(0)$ is not known. Fig.7. shows the correction results in the case of a) $\hat{y}_{2}(0)=0$, b) $\hat{y}_{2}(0)=0.5$. There can be seen the initial transient state (Fig.7b.) which disappears after a dozen or so steps of the algorithm realization. However, in the steady state a practically error free correction was achieved (errors at the level of $10^{-15}$ ).

a)

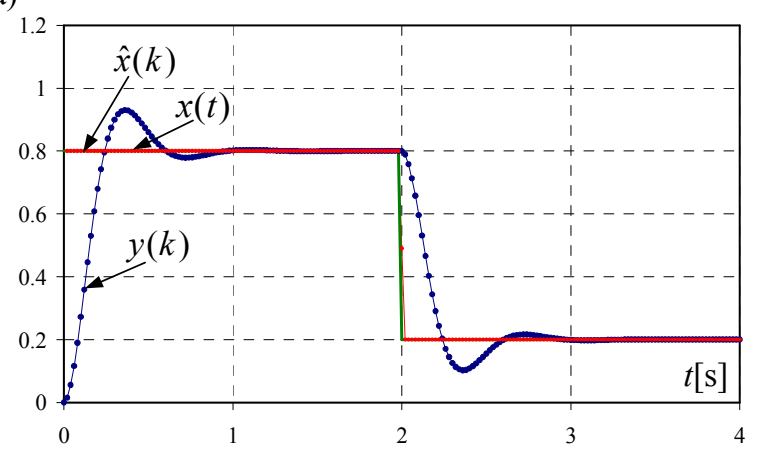

b)

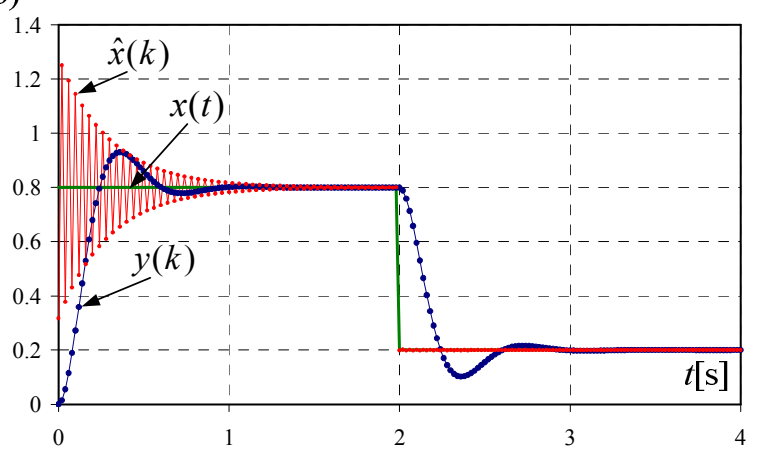

Fig.7. Results of the dynamic error correction performed by the recurrent neural network composed of two perceptrons (Fig.4.) for the initial value a) $\hat{y}_{2}(0)=0$, b) $\hat{y}_{2}(0)=0.5$. 
However, it should be mentioned that in both described cases, the ideal learning and testing sets obtained from (22) and (24) were used. Under real measurement conditions, the obtained data are burdened with various types of errors. Fig.8. shows the results of the dynamic error correction performed by the ideally learned network from Fig.4., to which inputs there is given the data quantized with a) 12-bit, b) 16-bit resolution.

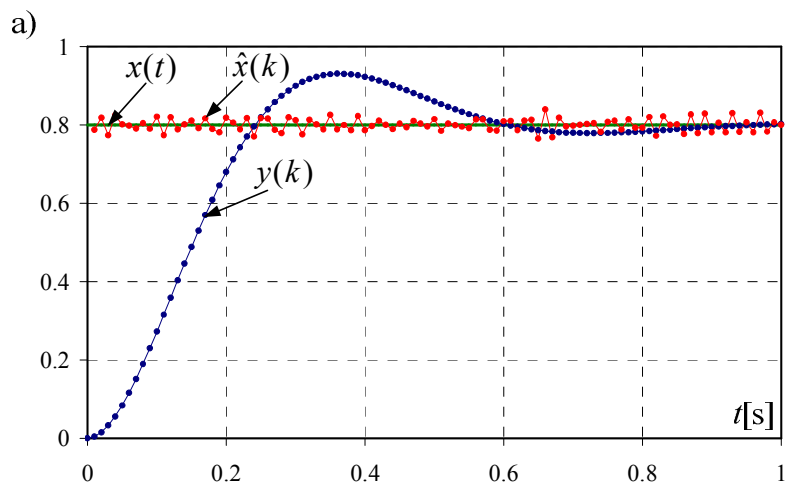

b)

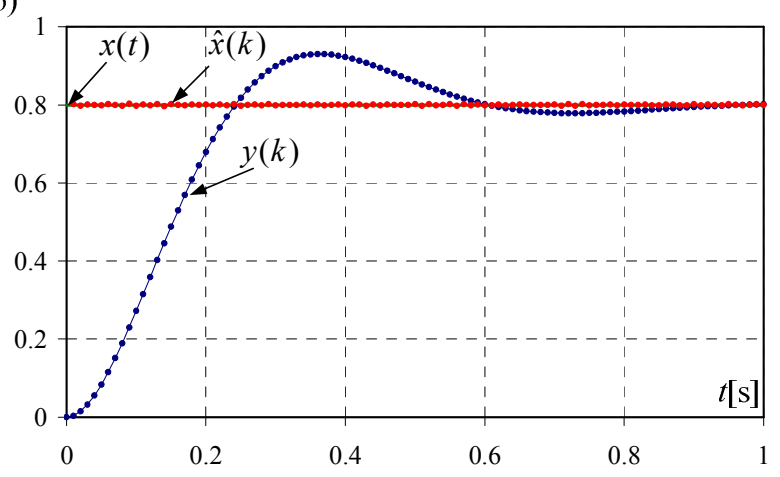

Fig.8. Results of the dynamic error correction in the case of the input data quantized with a) 12 bit b) 16 bit resolution.

\section{CONCLUDING REMARKS}

The simple recurrent ANN presented here enables the performing of the real-time dynamic error correction of transducers described in the general case by a linear differential equation of $n$-th order (1). It should be noted that the calculation of a single value of the instantaneous measurand requires only two samples of the transducer output waveform in transient state and $n-1$ values of the state variables from the previous correction step.

In the case of the second-order transducer, the real-time dynamic error correction can be performed in two ways. When using a recurrent chain of two linear perceptrons (Fig.4.) it is not necessary to know the subsequent values of state variable $\hat{y}_{2}$. In the first step it can be assumed that $\hat{y}_{2}(0)=0$. Wrong estimation of the $\hat{y}_{2}$ initial value causes occurrence of a transient state of the algorithm whose exemplary waveform is shown in Fig.7b. This type of operation can be used in the online, continuous dynamic error correction.
The second way is to use only one perceptron (Fig.5) and the online determination of the values of the state variable (derivative), which means non-recurrent operation. It should be noted that the use of the same method for determining state variable $\hat{y}_{2}$, both during perceptron learning and subsequent dynamic correction, allows avoiding the transient state. This solution, however, requires the use of an additional arithmetic circuit enabling current calculation of the value of state variable $\hat{y}_{2}$.

Under real measurement conditions, in which the obtained data is burdened with various types of errors, there should be taken into account the fact of random error reinforcement by dynamic error correction algorithms. It also refers to the neural implementation of such procedures, as illustrated in Fig.8. The values of the random errors at the network output can be reduced in several ways, e.g., by increasing the resolution of the $\mathrm{A} / \mathrm{D}$, increasing the sampling period $T_{d}$ or/and use of various random error filtering algorithms.

However, the use of ANN has other important advantages. For example, it is not necessary to identify transducer parameters and to determine dynamic correction algorithm coefficients. ANN performs these operations in the learning process. Parallel information processing by the ANN results in high speed of the correction performance. In addition, the described real-time neural dynamic error correction procedure can be used for the input signal of any course.

In a further stage of the research the developed ANN structure is expected to be complemented with additional neural elements enabling random error filtration.

\section{REFERENCES}

[1] Mukhopadhyay, S.C., Gupta, G.S. (2008). Smart Sensors and Sensing Technology. Lecture Notes in Electrical Engineering, Volume 20. Springer.

[2] Frank, R. (2000). Understanding Smart Sensors. Second edition. Artech House Inc.

[3] Morawski, R.Z. (1994). Unified approach to measurand reconstruction. IEEE Transactions on Instrumentation \& Measurement, 43 (2), 226-231.

[4] Roj, J. (2013). Metrological properties of RBF and MLP neural networks used for static errors correction in a sampling transducer. Przegląd Elektrotechniczny, 89 (1a), 84-87. (in Polish)

[5] Jakubiec, J., Makowski, P., Roj, J. (2009). Error model application in neural reconstruction of nonlinear sensor input signal. IEEE Transactions on Instrumentation and Measurement, 58 (3), 649-656.

[6] Roj, J. (2013). Neural Reconstruction of Measurement Signals. Gliwice, Wydawnictwo Politechniki Śląskiej. (in Polish)

[7] Haykin, S. (2008). Neural Networks: A Comprehensive Foundation. Third edition. Prentice Hall.

[8] Gupta, M., Homma, N., Jin, L. (2003) Static and Dynamic Neural Networks: From Fundamentals to Advanced Theory. John Wiley \& Sons.

[9] Bishop, Ch.M. (1996). Neural Networks for Pattern Recognition. Oxford University Press. 
[10] Jackowska-Strumiłło, L. (2001). Dynamic temperature error correction by the use of artificial naural networks. In TEMPMEKO 2001 : 8th International Symposium on Temperature and Thermal Measurements in Industry and Science, 19-21 June 2001. Berlin: VDE-Verlag, 1097-1102.

[11] Wu, D., Huang, S., Zhao, W., Xin, J. (2009). Infrared thermometer sensor dynamic error compensation using Hammerstein neural network. Sensors and Actuators A: Physical, 149 (1), 152-158.

[12] Hornik, K., Stinchcombe, M., White, H. (1989). Multilayer feedforward networks are universal approximators. Neural Networks, 2, 359-366.

[13] Feldkamp, L.A., Puskorius, G.V. (1998). A signal processing framework based on dynamic neural networks with application to problems in adaptation, filtering, and classification. Proceedings of the IEEE, 86 (11), 2259-2277.

[14] Park, J., Sandberg, I.W. (1991). Universal approximation using radial-basis-function networks. Neural Computation, 3 (2), 246-257.

[15] Singh, A.P., Kamal, T.S., Kumar, S. (2006). Development of a virtual linearizer for correcting transducer static nonlinearity. ISA Transactions, 45 (3), 319-328.

[16] Patra, J.C., Chakraborty, G., Meher, P.K. (2008). Neural-network-based robust linearization and compensation technique for sensors under nonlinear environmental influences. IEEE Transactions on Circuits and Systems I: Regular Papers, 55 (5), 13161327.

[17] Wang, Y.-X, Li, Z.-H., Zhang, T.-H. (2010). Research of ultrasonic flow measurement and temperature compensation system based on neural network. In International Conference on Artificial Intelligence and Computational Intelligence (AICI 2010), 23-24 October 2010. IEEE, Vol. 1, 268-271.

[18] Jackowska-Strumiłło, L., Urbański, M. (2001). Linearisation of thermistor characteristic by the use of artificial naural networks. In TEMPMEKO 2001 : 8th International Symposium on Temperature and Thermal Measurements in Industry and Science, 19-21 June 2001. Berlin: VDE-Verlag, 1035-1040.

[19] Massicotte, D., Legendre, S., Barwicz, A. (1998). Neural-network-based method of calibration and measurand reconstruction for a high-pressure measuring system. IEEE Transactions on Instrumentation and Measurement, 47 (2), 362-370.

[20] Morawski, R.Z., Miękina, A., Wiśniewski, M., Barwicz, A. (2002). Neural network based calibration of a mini-spectrophotometer. In Proceedings of the 19th IEEE Instrumentation and Measurement Technology Conference (IMTC 2002). IEEE, Vol. 2, 1083-1086.

[21] Rath, S.K., Patra, J.C., Kot, A.C. (2000). An intelligent pressure sensor with self-calibration capability using artificial neural networks. In IEEE International Conference on Systems, Man, and Cybernetics. IEEE, Vol. 4, 2563-2568.
[22] Nabielec, J. (1999). An outlook on the DSP dynamic error blind correction of the analog part of the measurement channel. In Proceedings of the 16th IEEE Instrumentation and Measurement Technology Conference (IMTC '99). IEEE, Vol. 2, 709-712.

[23] Morawski, R.Z. (2007). On teaching measurement applications of digital signal processing. Measurement, 40 (2), 213-223.

[24] Wu, J., Zhang, Z., Dong, G., Wang, W. (2011). Realtime correction for sensor's dynamic error based on DSP. In IEEE Instrumentation and Measurement Technology Conference (I2MTC), 10-12 May 2011. IEEE, 1-6.

[25] Wu, J., Zhang, Z., Wang, W. (2012). FPGA implementation of high speed parallel correction for sensor's dynamic error. Chinese Journal of Sensors and Actuators, 25 (1), 67-71.

[26] Jafaripanah, M., Al-Hashimi, B.M, White, N.M. (2005). Application of analog adaptive filters for dynamic sensor compensation. IEEE Transaction On Instrumentation and Measurement, 54 (1), 245-251.

[27] Jafaripanah, M., Al-Hashimi, B.M, White, N.M. (2003). Load cell response correction using analog adaptive techniques. In International Symposium on Circuits and Systems (ISCAS2003), 25-28 May 2003. IEEE, IV-752-IV-755.

[28] Bahar, H.B., Safari, E., Ghazijahani, H.A. (2010). Artifcial neural networks based dynamicweight estimation with optical arrangement. Transactions D: Computer Science \& Engineering and Electrical Engineering, 17 (2), 189-195.

[29] Bahar, H.B., Horrocks, D.H. (1998). Dynamic weight estimation using an artificial neural network. Artificial Intelligence in Engineering, 12 (1-2), 135-139.

[30] Fraden, J. (2010). Handbook of Modern Sensors. Springer.

[31] Burns, R.S.(2001). Advanced Control Engineering. Butterworth-Heinemann.

[32] Bojarska, M., Jakubiec, J. (2001). A method of modelling sampling converter dynamic errors. Metrology and Measurement Systems, 8 (4), 337-355.

[33] Palm, III, W.J. (2010). System Dynamics. Second edition. McGraw-Hill.

[34] Dorf, R.C., Bishop, R.H. (2008). Modern Control Systems. Eleventh edition. Prentice Hall.

[35] Jakubiec, J. (2010). Data Errors and Uncertainties in the Measurement and Control Systems. Gliwice, Wydawnictwo Politechniki Śląskiej. (in Polish)

[36] MathWorks Inc. Neural Network Toolbox: User's Guide.

[37] Hagan, M.T., Menhaj, M. (1994). Training feedforward networks with the Marquardt algorithm. IEEE Transactions on Neural Networks, 5 (6), 989993.

Received April 8, 2013. Accepted November 21, 2013. 